\title{
Sexual And Reproductive Health Needs Of Street-Involved Young People: Findings From A Baseline Survey In Southwest Nigeria
}

Atinuke 0 Olaleye ( $\nabla$ tinukeolaleye@gmail.com )

Babcock University https://orcid.org/0000-0003-4935-3926

\author{
Mary O. Obiyan \\ Obafemi Awolowo University \\ Morenike O. Folayan \\ Obafemi Awolowo University
}

\section{Research}

Keywords: Street youth, Street children, Contraception, Adolescents, Sexual and Reproductive Health, Nigeria

Posted Date: February 18th, 2020

DOI: https://doi.org/10.21203/rs.2.23753/v1

License: (c) This work is licensed under a Creative Commons Attribution 4.0 International License. Read Full License 


\section{Abstract}

Background To achieve the Sustainable Development Goal 3, street-involved young people (SIYP) require consideration in the strategies for ensuring universal access to sexual and reproductive healthcare. This study aims to determine the ageand sex-specific sexual and reproductive health (SRH) needs of SIYP in Nigeria.

Methods This was a cross-sectional study that recruited 1,505 SIYP aged 10-24 years using respondent-driven and timelocation sampling. Data were collected on socioeconomic characteristics, access to SRH information, contraceptive knowledge and use, sexual behavior and sexual practice using interviewer-administered questionnaires. The outcome variables were inconsistent condom use, multiple sexual partners and transactional sex. Binomial regression analysis models were developed to determine risk indicators for outcome variables.

Results Though 968 (64.3\%) participants were sexually active and 1,089 (72.4\%) knew about modern contraception, only 300 (31.0\%) sexually active respondents use modern contraceptives. Knowledge of modern contraception (AOR: 0.11; 95\% C.I: $0.01-0.82, p=0.03$ ) and being employed (AOR: 0.38; 95\% C.I: 0.15-0.95, $p=0.04$ ) reduced the odds for inconsistent condom use among male SIYPs. For the female SIYPs, knowledge of modern contraception reduced the odds for inconsistent condom use (AOR: 0.26 ; $95 \%$ C.I: 0.08-0.90, $p=0.03$ ), while having access to SRH information significantly increased the odds for inconsistent condom use (AOR: 5.06; 95\% C.I: 1.67-15.37, p=0.004).

Conclusion There are age- and sex- related differences in the factors associated with risky sexual behaviors among SIYP. Addressing these differences in the delivery of targeted interventions to reduce sexual health risk of SIYP may be required.

\section{Plain English Summary}

Street-involved young people (SIYP) are a vulnerable segment of the population that require consideration in ensuring universal access to sexual and reproductive healthcare. This study was conducted among 1,505 SIYP in southwest Nigeria to determine their age- and sex- specific sexual and reproductive health (SRH) needs. Using intervieweradministered questionnaires, information was obtained on the socioeconomic characteristics, access to SRH information, contraceptive knowledge and use, sexual behavior and sexual practice of the respondents. Sexual risk behavior included inconsistent condom use, multiple sexual partners and transactional sex. Findings from the study showed that despite a relatively high awareness of modern contraception, less than a third of sexually active SIYP use modern contraceptives. There were also age and sex differences in the sexual risk behaviors of SIYP. These findings imply that SIYP need age- and sex- segmented targeted interventions to address their sexual and reproductive health needs.

\section{Background}

More than half of the global population are young people age 10-24 years [1]. Young people have an innate curiosity and are adventurous, thus making it critical to address their sexual and reproductive health (SRH) needs. Access to information to enable them make informed choices about their sexuality and protect them from unwanted pregnancies, sexually transmitted infections and its sequelae is critical [2]. Access of adolescents and young persons to culturally sensitive messages on $\mathrm{SRH}$ is still inadequate, as there is limited context-specific evidence to facilitate the development of culturally appropriate information for the diverse populations of young people $[3,4]$.

To facilitate the development of strategic actions for the non-homogenous population of young persons, appropriate segmentation of the population based on their social context and their vulnerability is necessary $[5,6]$. Population segmentation facilitates the design and implementation of programs that enhance equitable access to services. A vulnerable segment of young people are street-involved young people (SIYP), described as those who live "on the street" (work on the streets but return home at night or maintain contact with families) and those who live "of the street" (those who never return home or have lost contact with families) [7, 8]. A street child is: "Any girl or boy who has not reached 
adulthood, for whom the street in the widest sense of the word, including unoccupied dwellings, wasteland, and so on, has become his or her habitual abode and/or source of livelihood, and who is inadequately protected, directed, and supervised by responsible adults" [9]. Several millions of children and youths are street-connected, with the largest burden in low and middle-income countries [10-12].

SIYP have limited understanding of and access to information on $\mathrm{SRH}$, making it challenging to make healthy choices and thereby increasing their prospect for having risky sexual behavior such as unprotected sexual intercourse, early age of sexual debut, having multiple sexual partners, and engaging in transactional sex $[13,14]$.

The psychosocial framework for understanding adolescent risk behavior described by Jessor [15] helps our understanding of the complex interrelationship of the risk (and protective) factors that influence risk behavior and consequent health or life outcomes of adolescents. Risky sexual behavior of young people has been described at the personal, family, peer, school and community levels [16]. Personal factors such as age, level of education, sexual knowledge and sources of sex information have been implicated. Family structure and relationships, peer influence, religious beliefs, economic constraints and social changes in the community also influence sexual decision-making among adolescents [16, 17]. Poor access of adolescents and young persons to SRH services because of social stigmatization and marginalization makes it challenging for SIYP to access SRH-related treatment, with attendant complications $[13,18]$. These SRH-related risk factors fit into four of the five domains described by Jessor [15] namely: the social environment, the perceived environment, personality, other behavior and biology/genetics.

SIYP are often hidden and thus neglected in most public health interventions [19]. SIYP therefore bear a presumably more substantial burden of SRH needs, including unintended pregnancy and unsafe abortion. One of the specific targets of the Sustainable Development Goal 3 is that by 2030, countries should ensure universal access to SRH-care services, including access to family planning, information and education and the integration of reproductive health into national strategies and programs [20]. To meet this goal, it is important to understand how the interplay of social, economic and cultural factors influence the sexual behaviors of young people, including SIYP. This study is an effort to address one of these gaps: to determine age- and sex-specific SRH needs of SIYP in Nigeria.

\section{Methods}

Study design, population and study sites

This is a cross-sectional study conducted to determine the SRH needs of SIYPs in two states in southwestern Nigeria. It was a follow-up to the water access, sanitation and hygiene community engagement program reported by Folayan et al [21]. The two States - Lagos and Osun - were purposively selected based on their level of industrialization. Lagos is an industrialized cosmopolitan State while Osun is a less industrialized State.

The study population were male and female adolescents aged 10 to 24 years, living 'on' and 'of' the street. At each study location, places where SIYPs aggregate in large numbers such as major streets, market places and motor-parks were identified through a mapping exercise conducted by the research team along with officials of the State Ministry of Health. These locations were grouped as clusters. The clusters in Lagos State were Bariga and Ajah while that for Osun State were Oke-Baale, Olaiya and Sabo.

\section{Sample size}

The determination of sample size for this study was guided by Turner [22] recommended estimates to derive sample size for surveys on orphaned and vulnerable children (OVC) in homeless situations. Considering the unavailability of data to generate a prevalence rate of OVC in the proposed study environment, the suggested minimum sample size of 800 to 1000 was adopted and 1,505 street-involved young people were enrolled in this study.

Study recruitment procedure: Following community engagement, the study participants were recruited using respondentdriven sampling and time-location sampling methods $[23,24]$. The respondent-driven sampling developed by Heckathorn

Page 3/18 
[25] is a sampling process whereby participants recruit their peers, in hard-to-reach study populations [26]. In this study, the first ten seeds recruited through the respondent-driven sampling were given a labelled tag with generated identity numbers. After their enrollment, they were given two additional tags to recruit friends/peers. Each referred respondent was checked for eligibility, enrolled and interviewed once the eligibility criteria were met. The eligibility criteria were: aged 10-24 years, living 'on' or 'of' the street in Lagos or Osun State and mentally stable.

Study participant recruitment through respondent-driven sampling was slow, as there were identified boundaries within street groups and low density of social networks. The research team then adopted time-location sampling to recruit the target population at specific times, days and venues where SIYP gather [27]. To limit selection bias with this method, we ensured that the venue-day-time options with possible large turnouts of SIYP were selected for the recruitment of participants.

Through the respondent-driven sampling, 34 seeds were recruited, and 465 coupons were given out over six recruitment waves within five days. Only 120 respondents were recruited and interviewed through this method. In contrast, the timelocation sampling method resulted in 1,800 coupons given, and 1,385 respondents recruited and interviewed [See Supplementary File 1 for study recruitment characteristics].

The questionnaire was administered in a place the respondent identified to be most comfortable to respond to questions. Data were collected by the field worker electronically using REDcap - a secure web application used to build and manage online surveys/databases.

Study instrument

The research instrument was adapted from the "Illustrative questionnaire for interview-surveys with young people" designed by John Cleland for the World Health Organisation [28]. The content of the questionnaire was revised by two experts in sexual and reproductive health, to address the study objectives and fit within the Nigerian cultural context. The revised questionnaire was pre-tested with 20 purposively selected SIYPS at two locations aside the study sites (Ile-Ife and Ibadan), to ascertain the clarity and conciseness of the questions. The tool was revised for language and procedural clarity, then translated into the local dialect (Yoruba) for respondents that do not understand English language. Field workers that speak the other national languages - Ibo and Hausa - were made available to interpret key concepts to study participants where necessary.

Measurement of variables

The explanatory variable in this study was 'knowledge of SRH' measured by (i) knowledge of modern contraceptives; and (ii) access to SRH information. A knowledge of modern contraceptives was deduced from responses to a question asked from respondent - "do you know of any of these methods which men and women can use to prevent pregnancy?" There were seven options - (i) injection, (ii) condom, (iii) emergency contraception, (iv) traditional method, (v) withdrawal method, (vi) safe period and (vii) periodic abstinence. Options i-iii were recoded as ' 1 ' implying knowledge of modern method of contraception; and others were recoded as ' 0 '.

Further, respondents were asked 'if they ever attended/or were given a talk on SRH'; a positive response was assigned "1", and otherwise " 0 ". The respondents were also asked to state the contraceptive method used at last sexual activity by self or partner.

The variables adjusted for in this study were selected background characteristics of respondents: age [10-14, 15-19, 2024years], educational status [none, primary, secondary] and employment status [not working, working]. The outcome variable was 'sexual risk behavior' proxied by three variables: (i) inconsistent use of condom, (ii) multiple sexual partners; and (iii) transactional sex. Both male and female respondents who were sexually active were asked if they used condom at the last sexual activity. An answer in the affirmative was assigned " 1 "and a negative response was assigned " 0 ". Respondents were also asked the number of sex partners they currently had. Those who responded they had one were assigned " 1 " while those with more than one sex partner were assigned " 0 ". Also, two questions were asked to probe for 
transactional sex: "have you ever paid or exchange gift for sex?", and "have you ever been paid or receive gift in exchange for sex?". An answer in the affirmative to either of the questions was assigned " 1 " and a negative response was assigned "0".

\section{Analysis}

Data analysis was conducted using Stata SE 15.1 (Stata Corporation, College Station, Texas). The univariate analysis was conducted to determine the percentage distribution of participants by age in grouped years [10-14, 15-19, 20-24], sex, educational level, employment status, knowledge of modern contraception and access to SRH information. Bivariate analysis was conducted to test associations between the explanatory and outcome variables using Pearson chi-square test. The inferential analysis was conducted using logistic regression to determine the risk indicators for the outcome variables by sex. Two models guided the regression analysis. The first model regressed each of the outcomes against the explanatory variables, while the second model adjusted for confounders (age, literacy level, and work status). Based on the Pearson chi-square significant association test, we set the $p$-value cut-off point of $0.20(p \leq 0.20)$ for the inclusion of confounders in the regression model. The Hosmer-Lemeshow goodness-of-fit test was conducted to ascertain all study variables fulfilled the underlying assumption of a univariate regression. Statistical significance was considered at p-value less or equal to 0.05 .

\section{Results}

Background characteristics of study participants The distribution of the respondents by age, level of education, and work status is shown in Table 1. Most of the respondents were in the 15-19 years age group (41.3\%), had no formal education (50.8\%) and were unemployed at the time of the interview (57.2\%).

Knowledge of sexual and reproductive health

Table 2 also shows the age and sex distribution of respondents by their knowledge about SRH issues. Most (72.4\%) of the respondents knew about modern methods of contraception, though the majority (84.0\%) had no access to SRH information. Those that had access to information (16\%) received it through formal talks or at health facilities. More female than male respondents knew about modern contraceptive methods (75.9\% vs $69.8 \% ; p=0.01)$ and had access to SRH information $(21.6 \%$ vs $11.9 \%$; $p<0.001)$. Also, significantly more SIYPs who were $10-14$-years-old did not know about modern contraception $(p<0.001)$ and had no access to SRH information $(p<0.001)$ when compared with those 1519years-old and 20-24years-old. 
Table 2

Sexual Health Practices among sexually active SIYP by Sex in South-West, Nigeria $(\mathrm{N}=968)$

\begin{tabular}{|c|c|c|c|c|}
\hline Variables & $\begin{array}{l}\text { Male (N = 548) } \\
n(\%)\end{array}$ & $\begin{array}{l}\text { Female }(\mathrm{N}=420) \\
\mathrm{N}(\%)\end{array}$ & $x^{2} ; p$ value & $\begin{array}{l}\text { Total }(N=968) \\
(\%)\end{array}$ \\
\hline \multicolumn{5}{|c|}{ Contraceptive Method used at last sexual activity* } \\
\hline Condom & $\begin{array}{l}130 \\
(23.7 \%)\end{array}$ & $\begin{array}{l}106 \\
(25.2 \%)\end{array}$ & \multirow[t]{5}{*}{$\begin{array}{l}x^{2}=1.99 \\
p=0.16\end{array}$} & $\begin{array}{l}236 \\
(23.1 \%)\end{array}$ \\
\hline Pills & $\begin{array}{l}12 \\
(2.2 \%)\end{array}$ & $\begin{array}{l}35 \\
(8.3 \%)\end{array}$ & & $\begin{array}{l}64 \\
(4.3 \%)\end{array}$ \\
\hline Withdrawal method & $\begin{array}{l}15 \\
(2.7 \%)\end{array}$ & $\begin{array}{l}19 \\
(4.5 \%)\end{array}$ & & $\begin{array}{l}30 \\
(1.9 \%)\end{array}$ \\
\hline Safe Period & $\begin{array}{l}6 \\
(1.1 \%)\end{array}$ & $\begin{array}{l}9 \\
(2.1 \%)\end{array}$ & & $\begin{array}{l}15 \\
(1.2 \%)\end{array}$ \\
\hline Traditional & $\begin{array}{l}1 \\
(0.1 \%)\end{array}$ & $\begin{array}{l}10 \\
(1.57 \%)\end{array}$ & & $\begin{array}{l}11 \\
(0.7 \%)\end{array}$ \\
\hline \multicolumn{5}{|l|}{ Multiple Partners } \\
\hline one sexual partner & $\begin{array}{l}222 \\
(40.5 \%)\end{array}$ & $\begin{array}{l}204 \\
(48.6 \%)\end{array}$ & \multirow[t]{2}{*}{$\begin{array}{l}x^{2}=6.27 \\
p<0.01\end{array}$} & $\begin{array}{l}426 \\
(28.3 \%)\end{array}$ \\
\hline more than one sexual partner & $\begin{array}{l}326 \\
(59.5 \%)\end{array}$ & $\begin{array}{l}216 \\
(51.4 \%)\end{array}$ & & $\begin{array}{l}542 \\
(36.0 \%)\end{array}$ \\
\hline \multicolumn{5}{|l|}{ Transactional Sex } \\
\hline No & $\begin{array}{l}409 \\
(74.6 \%)\end{array}$ & $\begin{array}{l}338 \\
(80.5 \%)\end{array}$ & \multirow[t]{2}{*}{$\begin{array}{l}x^{2}=4.60 \\
p=0.03\end{array}$} & $\begin{array}{l}747 \\
(77.2 \%)\end{array}$ \\
\hline Yes & $\begin{array}{l}139 \\
(25.4 \%)\end{array}$ & $\begin{array}{l}82 \\
(19.5 \%)\end{array}$ & & $\begin{array}{l}221 \\
(22.8 \%)\end{array}$ \\
\hline \multicolumn{5}{|l|}{ Inconsistent use of condom } \\
\hline No & $\begin{array}{l}25 \\
(4.6 \%)\end{array}$ & $\begin{array}{l}40 \\
(9.5 \%)\end{array}$ & \multirow[t]{2}{*}{$\begin{array}{l}x^{2}=9.34 \\
p<0.002\end{array}$} & $\begin{array}{l}65 \\
(6.7 \%)\end{array}$ \\
\hline Yes & $\begin{array}{l}523 \\
(95.4 \%)\end{array}$ & $\begin{array}{l}380 \\
(90.5 \%)\end{array}$ & & $\begin{array}{l}903 \\
(93.3 \%)\end{array}$ \\
\hline
\end{tabular}

Sexual and reproductive health behavior of sexually active SIYPS

Table 3 highlights the sex distribution of sexually active SIYPs by their sexual behavior. Of the 1,505 participants recruited, 968 (64.3\%) admitted to being sexually active. Of the sexually active SIYP, 300 (31.0\%) had ever used any form of modern contraception. The main form of contraception used was the condom. The majority (93.3\%) of sexually active SIYP used the condom inconsistently, more than half $(56.0 \%)$ had multiple sexual partners and $221(22.8 \%)$ engaged in transactional sex. More females than males reported ever using modern contraception $(p=0.27)$, used the condom inconsistently $(p<$ $0.002)$, had multiple sexual partners $(p<0.01)$, and engaged in transactional sex $(p<0.03)$. 
Table 3

Background Characteristics, SRH Knowledge and Sexual Risk Profile of Sexually Active SIYP by Sex (N = 968)

\begin{tabular}{|c|c|c|c|c|c|c|c|c|c|}
\hline \multirow[t]{3}{*}{ Variables } & \multicolumn{3}{|c|}{$\begin{array}{l}\text { Inconsistent Use of Condom } \\
(\mathrm{N}=903)\end{array}$} & \multicolumn{3}{|c|}{$\begin{array}{l}\text { Multiple Partners } \\
(\mathrm{N}=542)\end{array}$} & \multicolumn{3}{|c|}{$\begin{array}{l}\text { Transactional Sex } \\
(N=221)\end{array}$} \\
\hline & $\begin{array}{l}\text { Male } \\
(\mathrm{N}= \\
523)\end{array}$ & $\begin{array}{l}\text { Female } \\
(\mathrm{N}= \\
380)\end{array}$ & $\begin{array}{l}x^{2} \\
(p- \\
\text { value })\end{array}$ & $\begin{array}{l}\text { Male } \\
(\mathrm{N}= \\
326)\end{array}$ & $\begin{array}{l}\text { Female } \\
(\mathrm{N}= \\
216)\end{array}$ & $\begin{array}{l}x^{2} \\
(p- \\
\text { value })\end{array}$ & $\begin{array}{l}\text { Male } \\
(\mathrm{N}= \\
139)\end{array}$ & $\begin{array}{l}\text { Female } \\
\mathrm{N}=(82) \\
\mathrm{n}(\%)\end{array}$ & $\begin{array}{l}x^{2} \\
(p- \\
\text { value })\end{array}$ \\
\hline & $\mathrm{n}(\%)$ & $\mathrm{n}(\%)$ & & & n (\%) & & & & \\
\hline \multicolumn{10}{|l|}{ Age Group } \\
\hline $10-14$ years & $\begin{array}{l}98 \\
(18 . \\
7 \%)\end{array}$ & $\begin{array}{l}76 \\
(20.0 \%)\end{array}$ & \multirow{3}{*}{$\begin{array}{l}x^{2}= \\
8.49 \\
p= \\
0.01\end{array}$} & $\begin{array}{l}57 \\
(17.5 \%)\end{array}$ & $\begin{array}{l}46 \\
(21.3 \%\end{array}$ & \multirow{3}{*}{$\begin{array}{l}\mathrm{X}^{2}= \\
4.18 \\
p= \\
0.12\end{array}$} & $\begin{array}{l}15 \\
(10.8 \%)\end{array}$ & $\begin{array}{l}12 \\
(14.6 \%)\end{array}$ & \multirow{3}{*}{$\begin{array}{l}x^{2}= \\
7.56 \\
p= \\
0.02\end{array}$} \\
\hline 15-19 years & $\begin{array}{l}194 \\
(37.1 \%)\end{array}$ & $\begin{array}{l}179 \\
(47.1 \%)\end{array}$ & & $\begin{array}{l}120 \\
(36.8 \%)\end{array}$ & $\begin{array}{l}87 \\
(40.3 \%)\end{array}$ & & $\begin{array}{l}58 \\
(41.7 \%)\end{array}$ & $\begin{array}{l}38 \\
(46.3 \%)\end{array}$ & \\
\hline $20-24$ years & $\begin{array}{l}231 \\
(44.2 \%)\end{array}$ & $\begin{array}{l}125 \\
(32.9 \%)\end{array}$ & & $\begin{array}{l}149 \\
(45.7 \%)\end{array}$ & $\begin{array}{l}83 \\
(38.4 \%)\end{array}$ & & $\begin{array}{l}66 \\
(47.5 \%)\end{array}$ & $\begin{array}{l}32 \\
(39.0 \%)\end{array}$ & \\
\hline \multicolumn{10}{|c|}{ Level of Education } \\
\hline None & $\begin{array}{l}275 \\
(52.6 \%)\end{array}$ & $\begin{array}{l}170 \\
(44.8 \%)\end{array}$ & \multirow{2}{*}{$\begin{array}{l}x^{2}= \\
7.60 \\
p= \\
0.02\end{array}$} & $\begin{array}{l}164 \\
(50.3 \%)\end{array}$ & $\begin{array}{l}106 \\
(49.1 \%)\end{array}$ & \multirow{2}{*}{$\begin{array}{l}x^{2}= \\
6.17 \\
p= \\
0.05\end{array}$} & $\begin{array}{l}53 \\
(38.1 \%)\end{array}$ & $\begin{array}{l}31 \\
(37.8 \%)\end{array}$ & \multirow{2}{*}{$\begin{array}{l}x^{2}= \\
12.53 \\
p= \\
0.002\end{array}$} \\
\hline Primary & $\begin{array}{l}117 \\
(22.4 \%)\end{array}$ & $\begin{array}{l}95 \\
(25.0 \%)\end{array}$ & & $\begin{array}{l}73 \\
(22.4 \%)\end{array}$ & $\begin{array}{l}37 \\
(17.1 \%)\end{array}$ & & $\begin{array}{l}36 \\
(25.9 \%)\end{array}$ & $\begin{array}{l}25 \\
(30.5 \%)\end{array}$ & \\
\hline Secondary & $\begin{array}{l}131 \\
(25.1 \%\}\end{array}$ & $\begin{array}{l}115 \\
(30.3 \%)\end{array}$ & & $\begin{array}{l}89 \\
(27.3 \%)\end{array}$ & $\begin{array}{l}73 \\
(33.8 \%)\end{array}$ & & $\begin{array}{l}50 \\
(36.0 \%)\end{array}$ & $\begin{array}{l}26 \\
(31.7 \%)\end{array}$ & \\
\hline \multicolumn{10}{|c|}{ Employment status } \\
\hline Not working & $\begin{array}{l}270 \\
(51.6 \%)\end{array}$ & $\begin{array}{l}241 \\
(63.6 \%)\end{array}$ & \multirow{2}{*}{$\begin{array}{l}x^{2}= \\
3.56 \\
p= \\
0.06\end{array}$} & $\begin{array}{l}174 \\
(53.4 \%)\end{array}$ & $\begin{array}{l}130 \\
(60.5 \%)\end{array}$ & \multirow{2}{*}{$\begin{array}{l}\mathrm{X}^{2}= \\
0.06 \\
p= \\
0.81\end{array}$} & $\begin{array}{l}78 \\
(56.1 \%)\end{array}$ & $\begin{array}{l}54 \\
(67.5 \%)\end{array}$ & \multirow{2}{*}{$\begin{array}{l}x^{2}= \\
2.24 \\
p= \\
0.13\end{array}$} \\
\hline Working & $\begin{array}{l}253 \\
(48.4 \%)\end{array}$ & $\begin{array}{l}138 \\
(36.4 \%)\end{array}$ & & $\begin{array}{l}152 \\
(46.6 \%)\end{array}$ & $\begin{array}{l}85 \\
(39.5 \%)\end{array}$ & & $\begin{array}{l}61 \\
(43.9 \%)\end{array}$ & $\begin{array}{l}26 \\
(32.5 \%)\end{array}$ & \\
\hline \multicolumn{10}{|l|}{$\begin{array}{l}\text { Knowledge of } \\
\text { modern } \\
\text { contraceptives }\end{array}$} \\
\hline No & $\begin{array}{l}155 \\
(29.6 \%)\end{array}$ & $\begin{array}{l}83 \\
(21.8 \%)\end{array}$ & \multirow{2}{*}{$\begin{array}{l}x^{2}= \\
13.20 \\
p< \\
0.001\end{array}$} & $\begin{array}{l}88 \\
(27.0 \%)\end{array}$ & $\begin{array}{l}51 \\
(23.6 \%)\end{array}$ & \multirow{2}{*}{$\begin{array}{l}x^{2}= \\
0.27 \\
p= \\
0.60\end{array}$} & $\begin{array}{l}17 \\
(12.2 \%)\end{array}$ & $\begin{array}{l}10 \\
(12.2 \%)\end{array}$ & \multirow{2}{*}{$\begin{array}{l}x^{2}= \\
24.96 \\
p< \\
0.001\end{array}$} \\
\hline Yes & $\begin{array}{l}368 \\
(70.4 \%)\end{array}$ & $\begin{array}{l}297 \\
(78.2 \%)\end{array}$ & & $\begin{array}{l}238 \\
(73.0 \%)\end{array}$ & $\begin{array}{l}165 \\
(76.4 \%)\end{array}$ & & $\begin{array}{l}122 \\
(87.8 \%)\end{array}$ & $\begin{array}{l}72 \\
(87.8 \%)\end{array}$ & \\
\hline \multicolumn{10}{|l|}{$\begin{array}{l}\text { Access to SRH } \\
\text { information }\end{array}$} \\
\hline No & $\begin{array}{l}460 \\
(88.0 \%)\end{array}$ & $\begin{array}{l}287 \\
(75.5 \%)\end{array}$ & \multirow{2}{*}{$\begin{array}{l}\mathrm{X}^{2}= \\
0.15 \\
\mathrm{p}= \\
0.70\end{array}$} & $\begin{array}{l}284 \\
(87.1 \%)\end{array}$ & $\begin{array}{l}163 \\
(75.5 \%)\end{array}$ & \multirow{2}{*}{$\begin{array}{l}x^{2}= \\
0.13 \\
p= \\
0.72\end{array}$} & $\begin{array}{l}114 \\
(82.0 \%)\end{array}$ & $\begin{array}{l}59 \\
(72.0 \%)\end{array}$ & \multirow{2}{*}{$\begin{array}{l}\mathrm{x}^{2}= \\
4.21 \\
\mathrm{p}= \\
0.04\end{array}$} \\
\hline Yes & $\begin{array}{l}63 \\
(12.0 \%)\end{array}$ & $\begin{array}{l}93 \\
(24.5 \%)\end{array}$ & & $\begin{array}{l}42 \\
(12.9 \%)\end{array}$ & $\begin{array}{l}53 \\
(24.5 \%)\end{array}$ & & $\begin{array}{l}25 \\
(18.0 \%)\end{array}$ & $\begin{array}{l}23 \\
(28.0 \%)\end{array}$ & \\
\hline
\end{tabular}

Sexual behavior of SIYP by sex and age

Table 3 highlights the sex differences in the sexual risk profile of sexually active SIYPs, while Table 4 highlights the age differences. Significantly more male than female SIYP who were $20-24$ years old $(p<0.01)$, more females than males who were 15-19 years old $(p<0.01)$, and more females than males who knew about modern contraception $(p<0.001)$, 
used condom inconsistently. More 20-24-year-old males and 15-19-year-old females $(p<0.01)$ had multiple sex partners. Also, more male SIYP who were 20-24-years-old and more females 15-19-years-old ( $p=0.02$ ), more males who can read and write $(p=0.02)$, more 10-14-year-olds who cannot read and write $(p=0.02)$, and more 10-14-year-olds who had knowledge of modern contraception $(p<0.001)$ engaged in transactional sex. 
Table 4

Background Characteristics, SRH Knowledge and Sexual risk profile of sexually active SIYPs by Age Group (N=968)

\begin{tabular}{|c|c|c|c|c|c|c|c|c|c|}
\hline \multirow[t]{6}{*}{ Variables } & \multicolumn{3}{|c|}{$\begin{array}{l}\text { Inconsistent Use of Condom ( } \\
=903 \text { ) }\end{array}$} & \multicolumn{3}{|c|}{ Multiple Partners $(n=542)$} & \multicolumn{3}{|c|}{ Transactional Sex $(n=221)$} \\
\hline & $10-$ & $15-$ & $20-$ & $10-$ & $15-$ & $20-$ & $10-$ & $15-$ & $20-$ \\
\hline & 14 years & 19 years & 24 years & 14 years & 19 years & 24 years & 14 years & 19 years & 24 years \\
\hline & $(N=$ & $(N=$ & $(N=$ & $(N=$ & $(\mathrm{N}=$ & $(N=$ & $(\mathrm{N}=27)$ & $(N=96)$ & $(N=98)$ \\
\hline & 174) & 373) & 356) & 103) & 207) & 232) & $\mathrm{n}(\%)$ & $\mathrm{n}(\%)$ & $\mathrm{n}(\%)$ \\
\hline & $\mathrm{n}(\%)$ & $\mathrm{n}(\%)$ & $\mathrm{n}(\%)$ & $\mathrm{n}(\%)$ & $\mathrm{n}(\%)$ & $\mathrm{n}(\%)$ & & & \\
\hline \multicolumn{10}{|l|}{ Gender } \\
\hline Male & $\begin{array}{l}98 \\
(56.3 \%)\end{array}$ & $\begin{array}{l}194 \\
(52.0 \%)\end{array}$ & $\begin{array}{l}231 \\
(64.9 \%)\end{array}$ & $\begin{array}{l}57 \\
(55.3 \%)\end{array}$ & $\begin{array}{l}120 \\
(58.0 \%)\end{array}$ & $\begin{array}{l}149 \\
(64.2 \%)\end{array}$ & $\begin{array}{l}15 \\
(55.6 \%)\end{array}$ & $\begin{array}{l}58 \\
(60.4 \%)\end{array}$ & $\begin{array}{l}66 \\
(67.3 \%)\end{array}$ \\
\hline Female & $\begin{array}{l}76 \\
(43.7 \%)\end{array}$ & $\begin{array}{l}179 \\
(48.0 \%)\end{array}$ & $\begin{array}{l}125 \\
(35.1 \%)\end{array}$ & $\begin{array}{l}46 \\
(44.7 \%)\end{array}$ & $\begin{array}{l}87 \\
(42.0 \%)\end{array}$ & $\begin{array}{l}83 \\
(35.8 \%)\end{array}$ & $\begin{array}{l}12 \\
(44.4 \%)\end{array}$ & $\begin{array}{l}38 \\
(39.6 \%)\end{array}$ & $\begin{array}{l}32 \\
(32.7 \%)\end{array}$ \\
\hline$x^{2} ; p$ value & \multicolumn{3}{|c|}{$9.34 ; p=0.002$} & \multicolumn{3}{|c|}{$6.27 ; p<0.01$} & \multicolumn{3}{|c|}{$4.60 ; p=0.03$} \\
\hline \multicolumn{10}{|c|}{ Level of Education } \\
\hline None & $\begin{array}{l}119 \\
(68.4 \%)\end{array}$ & $\begin{array}{l}176 \\
(47.2 \%)\end{array}$ & $\begin{array}{l}150 \\
(42.1 \%)\end{array}$ & $\begin{array}{l}74 \\
(71.8 \%)\end{array}$ & $\begin{array}{l}104 \\
(50.2 \%)\end{array}$ & $\begin{array}{l}92 \\
(39.7 \%)\end{array}$ & $\begin{array}{l}18 \\
(66.7 \%)\end{array}$ & $\begin{array}{l}38 \\
(39.6 \%)\end{array}$ & $\begin{array}{l}28 \\
(28.6 \%)\end{array}$ \\
\hline Primary & $\begin{array}{l}47 \\
(27.0 \%)\end{array}$ & $\begin{array}{l}84 \\
(22.5 \%)\end{array}$ & $\begin{array}{l}81 \\
(22.8 \%)\end{array}$ & $\begin{array}{l}25 \\
(24.3 \%)\end{array}$ & $\begin{array}{l}37 \\
(17.9 \%)\end{array}$ & $\begin{array}{l}48 \\
(20.7 \%)\end{array}$ & $\begin{array}{l}8 \\
(29.6 \%)\end{array}$ & $\begin{array}{l}25 \\
(26.0 \%)\end{array}$ & $\begin{array}{l}28 \\
(28.6 \%)\end{array}$ \\
\hline Secondary & $\begin{array}{l}8 \\
(4.6 \%)\end{array}$ & $\begin{array}{l}113 \\
(30.3 \%)\end{array}$ & $\begin{array}{l}125 \\
(31.1 \%)\end{array}$ & $\begin{array}{l}4 \\
(3.9 \%)\end{array}$ & $\begin{array}{l}66 \\
(31.9)\end{array}$ & $\begin{array}{l}92 \\
(39.7 \%)\end{array}$ & $\begin{array}{l}1 \\
(3.7 \%)\end{array}$ & $\begin{array}{l}33 \\
(34.4 \%)\end{array}$ & $\begin{array}{l}42 \\
(42.9 \%)\end{array}$ \\
\hline$x^{2} ; p$ value & \multicolumn{3}{|c|}{$3.02 ; p=0.08$} & \multicolumn{3}{|c|}{$0.69 ; p=0.41$} & \multicolumn{3}{|c|}{$5.93 ; p=0.02$} \\
\hline \multicolumn{10}{|c|}{ Employment Status } \\
\hline $\begin{array}{l}\text { Not } \\
\text { working }\end{array}$ & $\begin{array}{l}141 \\
(81.0 \%)\end{array}$ & $\begin{array}{l}222 \\
(59.7 \%)\end{array}$ & $\begin{array}{l}148 \\
(41.6 \%)\end{array}$ & $\begin{array}{l}88 \\
(85.4 \%)\end{array}$ & $\begin{array}{l}115 \\
(55.8 \%)\end{array}$ & $\begin{array}{l}101 \\
(43.5 \%)\end{array}$ & $\begin{array}{l}19 \\
(70.4 \%)\end{array}$ & $\begin{array}{l}63 \\
(65.6 \%)\end{array}$ & $\begin{array}{l}50 \\
(52.1 \%)\end{array}$ \\
\hline Working & $\begin{array}{l}33 \\
(19.0 \%)\end{array}$ & $\begin{array}{l}150 \\
(40.3 \%)\end{array}$ & $\begin{array}{l}208 \\
(58.4 \%)\end{array}$ & $\begin{array}{l}15 \\
(14.6 \%)\end{array}$ & $\begin{array}{l}91 \\
(44.2 \%)\end{array}$ & $\begin{array}{l}131 \\
(56.5 \%)\end{array}$ & $\begin{array}{l}8 \\
(29.6 \%)\end{array}$ & $\begin{array}{l}33 \\
(34.4 \%)\end{array}$ & $\begin{array}{l}46 \\
(47.9 \%)\end{array}$ \\
\hline$x^{2} ; p$ value & \multicolumn{3}{|c|}{$3.56 ; p=0.06$} & \multicolumn{3}{|c|}{$0.06 ; p=0.81$} & \multicolumn{3}{|c|}{$2.24 ; p=0.13$} \\
\hline \multicolumn{10}{|c|}{ Knowledge of modern contraceptives } \\
\hline No & $\begin{array}{l}74 \\
(42.5 \%)\end{array}$ & $\begin{array}{l}71 \\
(19.0 \%)\end{array}$ & $\begin{array}{l}93 \\
(26.1 \%)\end{array}$ & $\begin{array}{l}48 \\
(46.6 \%)\end{array}$ & $\begin{array}{l}138 \\
(8.4 \%)\end{array}$ & $\begin{array}{l}53 \\
(22.8 \%)\end{array}$ & $\begin{array}{l}8 \\
(29.6 \%)\end{array}$ & $\begin{array}{l}10 \\
(10.4 \%)\end{array}$ & $9(9.2 \%)$ \\
\hline Yes & $\begin{array}{l}100 \\
(57.5 \%)\end{array}$ & $\begin{array}{l}302 \\
(81.0 \%)\end{array}$ & $\begin{array}{l}263 \\
(73.9 \%)\end{array}$ & $\begin{array}{l}55 \\
(53.4 \%)\end{array}$ & $\begin{array}{l}169 \\
(81.6 \%)\end{array}$ & $\begin{array}{l}179 \\
(77.2 \%)\end{array}$ & $\begin{array}{l}19 \\
(70.4 \%)\end{array}$ & $\begin{array}{l}86 \\
(89.6 \%)\end{array}$ & $\begin{array}{l}89 \\
(90.8 \%)\end{array}$ \\
\hline$x^{2} ; p$ value & \multicolumn{3}{|c|}{$13.20 ; p<0.001$} & \multicolumn{3}{|c|}{$0.27 ; p=0.60$} & \multicolumn{3}{|c|}{$24.96 ; p<0.001$} \\
\hline
\end{tabular}




\begin{tabular}{|c|c|c|c|c|c|c|c|c|c|}
\hline \multirow[t]{2}{*}{ Variables } & \multicolumn{3}{|c|}{$\begin{array}{l}\text { Inconsistent Use of Condom (n } \\
=903 \text { ) }\end{array}$} & \multicolumn{3}{|c|}{ Multiple Partners $(n=542)$} & \multicolumn{3}{|c|}{ Transactional Sex $(n=221)$} \\
\hline & $\begin{array}{l}10- \\
14 \text { years } \\
(\mathrm{N}= \\
174) \\
\mathrm{n}(\%)\end{array}$ & $\begin{array}{l}15- \\
19 \text { years } \\
(\mathrm{N}= \\
373) \\
\mathrm{n}(\%)\end{array}$ & $\begin{array}{l}20- \\
24 \text { years } \\
(\mathrm{N}= \\
356) \\
\mathrm{n}(\%)\end{array}$ & $\begin{array}{l}10- \\
14 \text { years } \\
(\mathrm{N}= \\
103) \\
n(\%)\end{array}$ & $\begin{array}{l}15- \\
19 \text { years } \\
(\mathrm{N}= \\
207) \\
n(\%)\end{array}$ & $\begin{array}{l}20- \\
24 \text { years } \\
(\mathrm{N}= \\
232) \\
\mathrm{n}(\%)\end{array}$ & $\begin{array}{l}10- \\
14 \text { years } \\
(\mathrm{N}=27) \\
n(\%)\end{array}$ & $\begin{array}{l}15- \\
19 \text { years } \\
(\mathrm{N}=96) \\
\mathrm{n}(\%)\end{array}$ & $\begin{array}{l}20- \\
24 \text { years } \\
(\mathrm{N}=98) \\
\mathrm{n}(\%)\end{array}$ \\
\hline No & $\begin{array}{l}170 \\
(97.7 \%)\end{array}$ & $\begin{array}{l}304 \\
(81.5 \%)\end{array}$ & $\begin{array}{l}273 \\
(76.7 \%)\end{array}$ & $\begin{array}{l}99 \\
(96.1 \%)\end{array}$ & $\begin{array}{l}164 \\
(79.2 \%)\end{array}$ & $\begin{array}{l}184 \\
(79.3 \%)\end{array}$ & $(92.6 \%)$ & (80.2\%) & (72.5\%) \\
\hline Yes & $\begin{array}{l}4 \\
(2.3 \%)\end{array}$ & $\begin{array}{l}69 \\
(18.5 \%)\end{array}$ & $\begin{array}{l}83 \\
(23.3 \%)\end{array}$ & $\begin{array}{l}4 \\
(3.9 \%)\end{array}$ & $\begin{array}{l}43 \\
(20.8 \%)\end{array}$ & $\begin{array}{l}48 \\
(20.7 \%)\end{array}$ & $\begin{array}{l}2 \\
(7.4 \%)\end{array}$ & $\begin{array}{l}19 \\
(19.8 \%)\end{array}$ & $\begin{array}{l}27 \\
(27.5 \%)\end{array}$ \\
\hline$x^{2} ; p$ value & \multicolumn{3}{|c|}{$0.15 ; p=0.70$} & \multicolumn{3}{|c|}{$0.12 ; p=0.72$} & \multicolumn{3}{|c|}{$4.21 ; p=0.04$} \\
\hline
\end{tabular}

Indicators for sexual risk behavior for male and female SIYP: Tables 5 and 6 highlight the outcome of the logistic regression determining the risk indicators for the three sexual risk behaviors of male and female SIYPs. Among the male SIYPs, knowledge about modern contraception significantly reduced the odds for inconsistent use of condom when compared with no knowledge (OR: 0.10; $95 \% \mathrm{Cl}$ : 0.01-0.77; AOR: $0.11 ; 95 \%$ Cl: $0.01-0.82$ ). 
Table 5

Logistic Regression of sexual-risk behavior on Knowledge and SRH information access among sexually-active Male SIYP $(\mathrm{N}=548)$

\begin{tabular}{|c|c|c|c|c|c|c|c|c|c|c|c|c|}
\hline \multirow{3}{*}{$\begin{array}{l}\text { Variables } \\
\\
\begin{array}{l}\text { Knowledge of } \\
\text { modern } \\
\text { contraceptives }\end{array}\end{array}$} & \multicolumn{6}{|c|}{ Model I: (Crude Odds Ratio) } & \multicolumn{6}{|c|}{ Model II (Adjusted Odds Ratio) } \\
\hline & \multicolumn{2}{|c|}{$\begin{array}{l}\text { Inconsistent } \\
\text { Use } \\
\text { of Condom }\end{array}$} & \multicolumn{2}{|c|}{$\begin{array}{l}\text { Multiple } \\
\text { Partners }\end{array}$} & \multicolumn{2}{|c|}{$\begin{array}{l}\text { Transactional } \\
\text { Sex }\end{array}$} & \multicolumn{2}{|c|}{$\begin{array}{l}\text { Inconsistent } \\
\text { Use } \\
\text { of Condom }\end{array}$} & \multicolumn{2}{|c|}{$\begin{array}{l}\text { Multiple } \\
\text { Partners }\end{array}$} & \multicolumn{2}{|c|}{$\begin{array}{l}\text { Transactional } \\
\text { Sex }\end{array}$} \\
\hline & OR & $\begin{array}{l}\mathrm{Cl} \text { at } \\
95 \%\end{array}$ & OR & $\begin{array}{l}\text { C I at } \\
95 \%\end{array}$ & OR & $\begin{array}{l}\text { C I at } \\
95 \%\end{array}$ & AOR & $\begin{array}{l}\mathrm{Cl} \text { at } \\
95 \%\end{array}$ & AOR & $\begin{array}{l}\mathrm{Cl} \text { at } \\
95 \%\end{array}$ & AOR & $\begin{array}{l}\text { C I at } \\
95 \%\end{array}$ \\
\hline No & 1.00 & - & 1.00 & - & 1.00 & - & 1.00 & - & 1.00 & - & 1.00 & - \\
\hline Yes & 0.10 & $\begin{array}{l}0.01- \\
0.77 \text { * }\end{array}$ & 1.18 & $\begin{array}{l}0.80- \\
1.72\end{array}$ & 3.49 & $\begin{array}{l}2.01- \\
6.07 \text { * }\end{array}$ & 0.11 & $\begin{array}{l}0.01- \\
0.82 \text { * }\end{array}$ & 1.15 & $\begin{array}{l}0.77- \\
1.70\end{array}$ & 3.01 & $\begin{array}{l}1.71- \\
5.29 \text { * }\end{array}$ \\
\hline \multicolumn{13}{|l|}{$\begin{array}{l}\text { Access to SRH } \\
\text { information }\end{array}$} \\
\hline No & 1.00 & - & 1.00 & - & 1.00 & - & 1.00 & - & 1.00 & - & 1.00 & - \\
\hline Yes & 0.75 & $\begin{array}{l}0.27- \\
2.08\end{array}$ & 1.12 & $\begin{array}{l}0.65- \\
1.91\end{array}$ & 1.50 & $\begin{array}{l}0.87- \\
2.59\end{array}$ & 1.20 & $\begin{array}{l}0.38- \\
3.84\end{array}$ & 1.03 & $\begin{array}{l}0.58- \\
1.81\end{array}$ & 1.20 & $\begin{array}{l}0.67- \\
2.16\end{array}$ \\
\hline \multicolumn{13}{|l|}{ Age Group } \\
\hline $10-14$ years & & & & & & & 1.00 & - & 1.00 & - & 1.00 & - \\
\hline $15-19$ years & & & & & & & 0.69 & $\begin{array}{l}0.14- \\
3.42\end{array}$ & 1.00 & $\begin{array}{l}0.61- \\
1.66\end{array}$ & 1.77 & $\begin{array}{l}0.91- \\
3.44\end{array}$ \\
\hline $20-24$ years & & & & & & & 0.56 & $\begin{array}{l}0.12- \\
2.67\end{array}$ & 1.09 & $\begin{array}{l}0.67- \\
1.78\end{array}$ & 1.83 & $\begin{array}{l}0.94- \\
3.54\end{array}$ \\
\hline \multicolumn{13}{|l|}{$\begin{array}{l}\text { Level of } \\
\text { Education }\end{array}$} \\
\hline None & & & & & & & 1.00 & - & 1.00 & - & 1.00 & - \\
\hline Primary & & & & & & & 0.63 & $\begin{array}{l}0.22- \\
1.79\end{array}$ & 1.02 & $\begin{array}{l}0.66- \\
1.57\end{array}$ & 1.43 & $\begin{array}{l}0.86- \\
2.38\end{array}$ \\
\hline Secondary & & & & & & & 0.73 & $\begin{array}{l}0.27- \\
2.02\end{array}$ & 1.21 & $\begin{array}{l}0.77- \\
1.92\end{array}$ & 1.75 & $\begin{array}{l}1.06- \\
2.89 *\end{array}$ \\
\hline \multicolumn{13}{|l|}{$\begin{array}{l}\text { Employment } \\
\text { Status }\end{array}$} \\
\hline Not working & & & & & & & 1.00 & - & 1.00 & - & 1.00 & - \\
\hline Working & & & & & & & 0.38 & $\begin{array}{l}0.15- \\
0.95 \text { * }\end{array}$ & & & 0.64 & $\begin{array}{l}0.43- \\
0.98^{\star}\end{array}$ \\
\hline$*_{p}<0.05$ & & & & & & & & & & & & \\
\hline
\end{tabular}


Table 6

Logistic Regression of sexual-risk behavior on Knowledge and SRH information access among sexually-active Female $\operatorname{SIYP}(N=420)$

\begin{tabular}{|lllllll|}
\hline Variables & \multicolumn{2}{l}{ Model I: (Crude Odds Ratio) } & & \multicolumn{2}{l}{ Model II (Adjusted Odds Ratio) } \\
\cline { 2 - 5 } & Inconsistent & Multiple & Transactional & Inconsistent & Multiple & Transactional \\
Use of & Partners & Sex & Use of & Partners & Sex \\
Condom & & & Condom & & \\
\end{tabular}

\begin{tabular}{|c|c|c|c|c|c|c|c|c|c|c|c|c|}
\hline $\begin{array}{l}\text { Knowledge of } \\
\text { modern } \\
\text { contraceptives }\end{array}$ & OR & $\begin{array}{l}\mathrm{C} \text { I at } \\
95 \%\end{array}$ & OR & $\begin{array}{l}\mathrm{C} \text { I at } \\
95 \%\end{array}$ & OR & $\begin{array}{l}\mathrm{C} \text { I at } \\
95 \%\end{array}$ & AOR & $\begin{array}{l}\mathrm{C} \text { I at } \\
95 \%\end{array}$ & AOR & $\begin{array}{l}\text { C I at } \\
95 \%\end{array}$ & AOR & $\begin{array}{l}\text { C I at } \\
95 \%\end{array}$ \\
\hline No & 1.00 & - & 1.00 & - & 1.00 & - & 1.00 & - & 1.00 & - & 1.00 & - \\
\hline Yes & 0.26 & $\begin{array}{l}0.08- \\
0.86 \text { * }\end{array}$ & 0.65 & $\begin{array}{l}0.40- \\
1.05\end{array}$ & 2.01 & $\begin{array}{l}0.98- \\
4.12\end{array}$ & 0.27 & $\begin{array}{l}0.08- \\
0.92^{\star}\end{array}$ & 0.79 & $\begin{array}{l}0.47- \\
1.31\end{array}$ & 1.77 & $\begin{array}{l}0.85- \\
3.72\end{array}$ \\
\hline
\end{tabular}

Access to SRH

information

\begin{tabular}{|c|c|c|c|c|c|c|c|c|c|c|c|c|}
\hline No & 1.00 & - & 1.00 & - & 1.00 & - & 1.00 & - & 1.00 & - & 1.00 & - \\
\hline Yes & 2.16 & $\begin{array}{l}0.87- \\
5.34\end{array}$ & 1.21 & $\begin{array}{l}0.76 \\
- \\
1.91\end{array}$ & 1.21 & $\begin{array}{l}0.70- \\
2.10\end{array}$ & 5.06 & $\begin{array}{l}1.67- \\
15.30 *\end{array}$ & 1.17 & $\begin{array}{l}0.71- \\
1.90\end{array}$ & 1.11 & $\begin{array}{l}0.61- \\
2.02\end{array}$ \\
\hline
\end{tabular}

Age Group

\begin{tabular}{|c|c|c|c|c|c|c|}
\hline $10-14$ years & 1.00 & - & 1.00 & - & 1.00 & - \\
\hline $15-19$ years & 0.63 & $\begin{array}{l}0.17- \\
2.34\end{array}$ & 0.62 & $\begin{array}{l}0.35- \\
1.08\end{array}$ & 1.21 & $\begin{array}{l}0.57- \\
2.57\end{array}$ \\
\hline $20-24$ years & 0.32 & $\begin{array}{l}0.08- \\
1.26\end{array}$ & 0.97 & $\begin{array}{l}0.53- \\
1.78\end{array}$ & 1.43 & $\begin{array}{l}0.63- \\
3.26\end{array}$ \\
\hline \multicolumn{7}{|l|}{$\begin{array}{l}\text { Level of } \\
\text { education }\end{array}$} \\
\hline None & 1.00 & - & 1.00 & - & 1.00 & - \\
\hline Primary & 1.41 & $\begin{array}{l}0.51- \\
3.90\end{array}$ & 0.44 & $\begin{array}{l}0.26- \\
0.74^{\star}\end{array}$ & 1.64 & $\begin{array}{l}0.88- \\
3.04\end{array}$ \\
\hline Secondary & 0.48 & $\begin{array}{l}0.21- \\
1.08\end{array}$ & 0.88 & $\begin{array}{l}0.53- \\
1.41\end{array}$ & 1.18 & $\begin{array}{l}0.63- \\
2.24\end{array}$ \\
\hline \multicolumn{7}{|l|}{$\begin{array}{l}\text { Employment } \\
\text { Status }\end{array}$} \\
\hline Not working & 1.00 & - & 1.00 & - & 1.00 & - \\
\hline Working & 0.96 & $\begin{array}{l}0.45- \\
2.03\end{array}$ & - & - & 0.65 & $\begin{array}{l}0.37- \\
1.14\end{array}$ \\
\hline
\end{tabular}

For the male respondents, being employed reduced the odds of using condom inconsistently (AOR: 0.38; 95\% Cl: 0.150.95 ) and engaging in transactional sex (AOR: $0.64 ; 95 \% \mathrm{Cl}: 0.43-0.98)$ when compared with those that were unemployed. Factors associated with increased odds for sexual risk behavior were having knowledge of modern contraception and a 
secondary education. Male SIYPs who had knowledge of modern contraception (AOR: 3.01; 95\% C.I: 1.71-5.29) and who had secondary school education (AOR: 1.75; 95\% C.I: 1.06-2.89) had increased odds of engaging in transactional sex when compared with those with no knowledge and no education respectively.

For female respondents, knowledge about modern contraception reduced the odds for inconsistent condom use when compared with those having no such knowledge (OR: 0.26; 95\% C.I: 0.08-0.86; AOR: 0.27; 95\% C.I: 0.08-0.92). Having primary education reduced the odds of having multiple sexual partners compared to those with no education (AOR: 0.44; 95\% C.I: $0.26-0.74, p=0.002$ ). A factor associated with increased odds for sexual risk behavior was access to SRH information. Female SIYPs who had access to SRH information were five times more likely to use condom inconsistently when compared with those who had no access to SRH information (AOR: 5.06; 95\% Cl: 1.67-15.30).

\section{Discussion}

The study indicates that the age- and sex- related SRH risk indicators for SIYPs differed. The older the age group, the less likely female SIYPs were to use condom inconsistently and have multiple sexual partners; and the less likely male SIYPs were to use condom inconsistently. While knowledge about modern contraception and education level were risk indicators for male SIYPs to engage in transactional sex, they were not for female SIYPs. Access to SRH information was a risk indicator for inconsistent condom use by female SIYPs and not male SIYP.

One of the strengths of this study is the large sample of SIYP recruited for the study. This makes it possible to conduct a robust analysis. The study also recruited participants from different cluster areas in each state and from two states with different economic profiles, thereby reducing the risk for non-representation of study participants. The fewer positive responses on some variables however, resulted in wide confidence intervals. Also, the face-to-face interviews increased the risk for social desirability response bias [29]. Despite these limitations, the study highlights the age and sex differences in $\mathrm{SRH}$ intervention needs for SIYPs in Nigeria.

First, the study highlights that there is a high unmet need for contraception among SIYPs. The proportion of sexually active female SIYP not using any form of contraception was higher than the $48.4 \%$ national estimate of unmet contraception need among sexually active unmarried women [30], and higher than the $40.5 \%$ reported for $10-24$ year olds living in households in Sierra Leone [31]. Unmet need for contraception among adolescents in developing countries is high - more than $40 \%$ in most countries in sub-Saharan Africa [32] - with sex, marital status, age, education and environment variations [33,34]. This high unmet need for contraception - a rate higher than the national average - is an indication that critical attention needs to be paid to this large population of adolescents. The 2019 draft national adolescent health policy recognizes the need to attend to the SRH need of street-involved young people for the first time. However, the translation of this policy into programs and interventions for this population once the policy is ratified, remains to be seen.

Second, we found that the contraception option used by SIYP is highly limited to condoms. Similar observations had been made in an earlier study, and the reason adduced is that condoms are relatively inexpensive and accessible [35]. The large proportion of sexually active SIYPs who use condom inconsistently significantly reduces its usefulness in the prevention of unintended pregnancy in this population. As SIYP are already in a precarious situation of increased vulnerability to SRH problems, the occurrence of unintended pregnancy may likely lead to unsafe abortions with its attendant adverse consequences. It is therefore essential to actively promote the use of more effective, user-independent long-acting reversible contraception (LARC), such as intrauterine devices and implants, in sexually active SIYP that want to delay childbearing [36].

LARC requires a visit to the health facility for insertion and removal. The low utilization of clinics for uptake and use of contraception by adolescents and young persons in Nigeria [37-39] will likely be a barrier for LARC access by SIYP. Poor attention paid to the SRH needs of SIYP will further limit their use of healthcare services to address their SRH needs.

Page $13 / 18$ 
Where young persons access clinics for SRH services, the discordance between the national policy documents and hospital family planning protocols on LARC further reduces the chances of SIYP accessing LARC. In Nigeria, though the full range of contraceptive services is theoretically available, contraception counseling for young people is often limited to barrier methods, pills and emergency contraception [19]. Efforts are needed to promote not only access of adolescents and young persons to SRH services, but also access of SIYPs to these services. Opportunities should be created for the healthcare providers to screen and counsel SIYP on SRH issues to promote support for making healthy choices [40].

Third, this study identified significant age and sex differences in the sexual risk behavior of SIYP - more females inconsistently use condom, while more males engage in transactional sex. Also, more of the younger adolescents SIYPs engage in risky sexual behavior. This information is important for the design of targeted and segmented interventions for SIYP. Such interventions will best be conducted by adapting SRH risk-reduction messages for different age- and sexsegmented populations.

Despite the importance of the study findings, there are a few limitations. First, this study was conducted in two of the $36+$ 1 States in Nigeria. There are geographical and cultural variations in the sexual and reproductive lifestyles of Nigerians across the different states, so the study findings may not be representative of Nigeria though it is likely to be representative of states in Southwestern Nigeria. The study is also cross-sectional in nature thus, we could only determine risk indicators and not risk factors for the SRH needs of SIYP in the study populations. The findings are however not different from what is known in the field, and so interventions designed based on the study findings may be appropriate.

\section{Conclusion}

Despite the study limitations, it highlights that age and sex differences exist in the sexual risk behaviors of SIYP in Southwest Nigeria. There is a high unmet need for contraception and inconsistent condom use among this population. These findings imply that SIYP need targeted interventions for age- and sex-segmented populations to implement relevant programs that address the SRH needs of this population.

\section{Abbreviations}

AOR: Adjusted odds ratio; Cl: Confidence interval; OR: Odds ratio; OVC: Orphaned and vulnerable children; SIYP: Streetinvolved young people; SRH - Sexual and Reproductive Health

\section{Declarations}

Ethics approval and consent to participate

The ethical approval for this study was obtained from the Institute of Public Health Research Ethics Committee, Obafemi Awolowo University lle-Ife (IPHOAU/12/1133). Additionally, ethical approval was obtained from Osun State (OSHREC/PRS/569T/154) Health Research Ethics Committee, and, social approval (LSMH2695/11/260/T) from the Lagos State Ministry of health. The study was conducted in line with the declaration of Helsinki. Written consent was obtained from all study participants. Parental consent was waived for children below 18 years from all the ethics committees. This was because the study was non-invasive, the children were street kids, many being matured minors, and the associated challenges of locating parents for study approval purposes.

Consent for publication

Not applicable

Page $14 / 18$ 
Availability of data and materials

The datasets analysed during the current study are available from the corresponding author on reasonable request.

Competing interests

MOF is a sectional editor with the BMC Oral Health. The other authors declare that they have no competing interests.

Funding

This research was supported by the Consortium for Advanced Research Training in Africa (CARTA). CARTA is jointly led by the African Population and Health Research Center and the University of the Witwatersrand and funded by the Carnegie Corporation of New York (Grant No-B 8606.R02), Sida (Grant No:54100113), the DELTAS Africa Initiative (Grant No: 107768/Z/15/Z) and Deutscher Akademischer Austauschdienst (DAAD). The DELTAS Africa Initiative is an independent funding scheme of the African Academy of Sciences (AAS)'s Alliance for Accelerating Excellence in Science in Africa (AESA) and supported by the New Partnership for Africa's Development Planning and Coordinating Agency (NEPAD Agency) with funding from the Wellcome Trust (UK) and the UK government. The funders had no role in the design of the study, data collection, analysis and interpretation, or writing of the manuscript. The statements made and views expressed are solely the responsibility of the authors.

Authors' contributions

MOO designed the study. MOO and AOO collected the data. MOO conducted the data analysis. AOO developed the first draft of the manuscript. MOO and MOF contributed to the development of the full manuscript. AOO, MOO and MOF agreed to the final version of the manuscript and its submission for publication. All authors have read and approved the manuscript.

\section{Acknowledgement}

We acknowledge the contributions of the study participants who took the time to actively engage with us on this project. The contributions of Dr Macellina Y. ljadunola to the design and implementation of this study are also acknowledged.

\section{References}

1. Brhane T, Assefa B, Birhan N. Reproductive health behaviour of street youth and associated factors in Gondar city, Northwest Ethiopia. International Journal Med Biomed Res. 2014;3(1):28-37

2. From childhood to womanhood: meeting the sexual and reproductive health needs of adolescent girls. 2012. https://www.unfpa.org/sites/default/files/resource-pdf/EN-SRH\%20fact\%20sheet-Adolescent.pdf Accessed 10 May 2019

3. United Nations Youth Strategy: Youth 2030. Working with and for Young People. Office of the Secretary General's Envoy on Youth. New York, United Nations. 2018. https://www.un.org/youthenvoy/wp-content/uploads/2018/09/1800080_UN-Youth-Strategy _Web.pdf Accessed 13 April 2019

Page $15 / 18$ 
4. United Nations -Youth, Department of Economic and Social Affairs. World Program of Action for Youth. 2019. https://www.un.org/development/desa/youth/health-wpay.html Accessed 13 April 2019.

5. UNFPA Strategy on Adolescents and Youth: Towards realizing the full potential of adolescents and youth. 2013. https://www.unfpa.org/sites/default/files/resource-pdf/ UNFPA\%20Adolescents\%20and\%20Youth\%20Strategy.pdf Accessed 24 May 2019

6. World Health Organisation. Global Accelerated Action for the Health of Adolescents (AA-HA!): guidance to support country implementation. Geneva: World Health Organization. 2017. Licence: CC BY-NC-SA 3.0 IGO

7. Worksheet for the Regional Operating Plan for Abandoned and Street Children. 1985. UNICEF, Geneva

8. Mugove K, Lincoln H. Why Children Leave their Homes for the Streets? The Case of Harare. International Journal of Scientific and Research Publications. 2015;5(10):301-305

9. Inter-NGO Programme on Street Children and Street Youth. Sub-regional seminar for the Mediterranean, Marseilles, 24th-27th October 1983: summary of proceedings. The Programme, 1984

10. Children in an Urban World: the State of the World's Children 2012. UN Child Fund. 2012. https://www.unicef.org/sowc2012/pdfs/SOWC-2012-Main-Report_EN_21Dec2011.pdf Accessed 9 April 2019

11. Carr-Hill R. Missing millions and measuring development progress. World Dev 2013;46:30-44. doi: 10.1016/j.worlddev.2012.12.017

12. Woan J, Lin J, Auerswald C. The health status of street children and youth in low-and middle-income countries: a systematic review of the literature. J Adolesc Health 2013;53(3):314-21.

https://doi.org/10.1016/j.jadohealth.2013.03.013

13. Isiugo-Abanihe UC, Olajide R, Nwokocha E, Fayehun F, Okunola R, Akingbade R. Adolescent sexuality and life skills education in Nigeria: To what extent have out-of-school adolescents been reached? Afr J Reprod Health. 2015;19(1):101-11

14. Blahd WH, O’Connor HM. High-Risk Sexual Behaviour. British Columbia: HealthLink BC. 2015. https://www.healthlinkbc.ca/healthtopics/tw9064. Accessed 20 January 2020

15. Jessor R. Risk behavior in adolescence: A psychosocial framework for understanding and action. Developmental Review. 1992;12(4):374-390. https://doi.org/10.1016/0273-2297(92)90014-S

16. Alimoradi Z, Kariman N, Simbar M, Ahmadi F. Contributing Factors to High-Risk Sexual Behaviors among Iranian Adolescent Girls: A Systematic Review. Int J Community Based Nurs Midwifery. 2017;5(1): 2-12. PMID: 28097173; PMCID: PMC5219561

17. World Health Organization. Broadening the horizon: Balancing protection and risk for adolescents. 2001. Geneva, Switzerland: Department of Child and Adolescent Health and Development Family and Community Health. https://apps.who.int/iris/bitstream/handle/10665/67242/WHO_FCH_CAH_01.20.pdf Accessed 20 January 2020

18. Aransiola JO, Zarowsky C. Street children, human trafficking and human security in Nigeria: competing discourses of vulnerability and danger. Etude Population Africa. 2014;27(2):398-410. https://doi.org/10.11564/27-2-484

19. Population Reference Bureau. Youth Family Planning Policy Scorecard: Measuring Commitment to Effective Policy and Program Interventions. 2018. https://www.prb.org/global-youth-family-planning-index/ Accessed 12 April 2019

20. World Health Organisation. Sustainable Development Goals. 2019. https://www.who.int/sdg/targets/en/ Accessed 10 March 2019

21. Folayan MO, Obiyan MO, Olaleye AO. Association between water, sanitation, general hygiene and oral hygiene practices of street-involved young people in Southwest Nigeria. BMC Oral Health. 2020;20:32 https://doi.org/10.1186/s12903-020-1022-z

22. Turner AG. Guidelines for Sampling Orphans and Other Vulnerable Children. 2003. http://ovcsupport.org/wpcontent/uploads/Documents/Guidelines_for_Sampling_Orphans_and_other_Vulnerable_Children_ 
Full_Manual_Eng_1.pdf Accessed 14 May 2019

23. Gayet C, Fernández-Cerdeño A. Time Location Sampling and Respondent Driven Sampling: techniques implementation for monitoring concentrated HIV/AIDS epidemic in Mexico. https://iussp2009.princeton.edu/papers/93359 Accessed 11 December 2019

24. Tran HV, Le LV, Johnston LG, Nadol P, Van Do A, Tran HT, Nguyen TA. Sampling Males Who Inject Drugs in Haiphong, Vietnam: Comparison of Time-Location and Respondent-Driven Sampling Methods. J Urban Health. 2015;92(4):74457. doi: 10.1007/s11524-015-9966-z.

25. Heckathorn DD. Respondent-Driven Sampling: A New Approach to the Study of Hidden Populations. Social Problems. 1997;44(2):174-199 DOI: 10.2307/3096941

26. Sadler GR, Lee HC, Lim RSH, Fullerton J. Recruitment of hard-to-reach Population Subgroups via Adaptations of the Snowball Sampling Strategy. Nursing \& health sciences. 2010;12(3):369-374. doi: 10.1111/j.14422018.2010.00541.x.

27. Cai WD, Zhao J, Zhao JK, Raymond HF, Feng YJ, Liu J, McFarland W, et al. HIV prevalence and related risk factors among male sex workers in Shenzhen, China: results from a time-location sampling survey. Sex Transm Infect. 2010;86:15e20. doi:10.1136/sti.2009.037440

28. Cleland J. Illustrative questionnaire for interview-surveys with young people. Asking Young People About Sexual and Reproductive Behaviors Illustrative Core Instruments, Geneva: World Health Organization. 2001. https://www.who.int/reproductivehealth/topics/adolescence/sample_core_instruments.pdf Accessed 10 April 2018

29. Grimm P. Social Desirability Bias. Wiley Online Library. 2010. https://onlinelibrary.wiley.com/doi/pdf/10.1002/ 9781444316568.wiem02057 Accessed 21 May 2019

30. Nigeria Demographic and Health Survey [NDHS]. Key Indicators Report. National Population Commission. 2018. Federal Republic of Nigeria Abuja, Nigeria. ICF International Rockville, Maryland, USA.

31. Labat A, Medina M, Elhassein M, Karim A, Jalloh MB, Dramaix M et al. Contraception determinants in youths of Sierra Leone are largely behavioral. Reprod Health. 2018;15(1):66. doi: 10.1186/s12978-018-0504-9.

32. Hindin MJ, Fatusi AO. Adolescent Sexual and Reproductive Health in Developing Countries: An Overview of Trends and Interventions. International Perspectives on Sexual and Reproductive Health.2009;35(2):58-62.

https://www.jstor.org/stable/40233805 Accessed 30 May 2019

33. Munakampe MN, Zulu JM, Michelo C. Contraception and abortion knowledge, attitudes and practices among adolescents from low and middle-income countries: a systematic review. BMC health services research.

2018;18(1):909. doi:10.1186/s12913-018-3722-5

34. Chandra-Mouli V, McCarraher DR, Phillips SJ, Williamson NE, Hainsworth G. Contraception for adolescents in low and middle income countries: needs, barriers, and access. Reproductive health. 2014;11(1):1. doi:10.1186/1742-4755-11-1

35. Bankole A, Malarcher S. Removing barriers to adolescents' access to contraceptive information and services. Stud Fam Plann. 2010;41(2):117-124. doi: 10.1111/j.1728-4465. 2010. 00232.x.

36. Kavanaugh ML, Frohwirth L, Jerman J, Popkin R, Ethier K. Long-acting reversible contraception for adolescents and young adults: patient and provider perspectives. Journal of Pediatric and Adolescent Gynecology. 2013;26(2):86-95. doi:10.1016/j.jpag.2012.10.006

37. Abiodun O, Abiodun OO, Ani F, Sotunsa O. Sexual and Reproductive Health Knowledge and Service Utilization among Inschool Rural Adolescents in Nigeria. J AIDS Clin Res. 2016;7:6 doi: 10.4172/2155-6113.1000576

38. Onasoga OA, Afolayan JA, Asamabiriowei TF, Jibril UN, Imam AA. Adolescents' Knowledge, Attitude and Utilization of Emergency Contraceptive Pills in Nigeria's Niger Delta Region. International Journal of MCH and AIDS. 2016;5(1):5360. DOI: 10.21106/ijma.93

39. Odo AN, Samuel ES, Nwagu EN, Nnamani PO, Atama CS. Sexual and reproductive health services (SRHS) for adolescents in Enugu state, Nigeria: a mixed methods approach. BMC health services research. 2018;18(1):92.

Page $17 / 18$ 
doi:10.1186/s12913-017-2779-x

40. McNicholas C, Peipert JF. Long-acting reversible contraception for adolescents. Current Opinion in Obstetrics \& Gynecology. 2012;24(5):293-298. doi:10.1097/GC0.0b013e32835686d5

\section{Supplementary Files}

This is a list of supplementary files associated with this preprint. Click to download.

- SupplementaryFile1BMCRH.docx

- STROBEchecklistSRHmanuscriptBMCRH.doc 